\title{
Study of Blood Flow with Effects of Slip in Arterial Stenosis Due to Presence of Transverse Magnetic Field
}

\author{
Sarfraz Ahmed \\ Department of Mathematics Jorhat engineering college. College,Jorhat , Assam- 785001 (India) \\ e-mail : sarfrazahmed.jrt@gmail.com
}

\begin{abstract}
The flow of blood in human circulatory system can be controlled by applying appropriate magnetic field. It is also well known that non-Newtonian nature of blood significantly influences the flows, particularly in the cases where blood vessels are curved, branching or narrow etc. Stenosis refers to localized narrowing of an artery and is a frequent result of arterial disease and is caused mainly due to intravascular atherosclerotic plaque which develops at the arterial wall and protrudes into the lumen of the vessel. Such constrictions disturb normal blood flow through the artery. Here study is made on the flow of blood through a stenosed artery with the effect of slip at the boundary in presence of transverse magnetic field considering blood as Casson fluid (non- Newtonian fluid). The equations of motion has have been solved numerically. The effect of various parameters on the flow characteristics like Hartmann number, Reynolds number has been discussed. Numerical results were obtained for different values of the Hartmann number $M$ and Reynolds number Re. It is observed that the fluid velocity decreases as the Hartmann number increases.
\end{abstract}

\section{KEYWORDS}

Magnetic field, Reynolds number, Hartmann number, Casson fluid, Blood flow

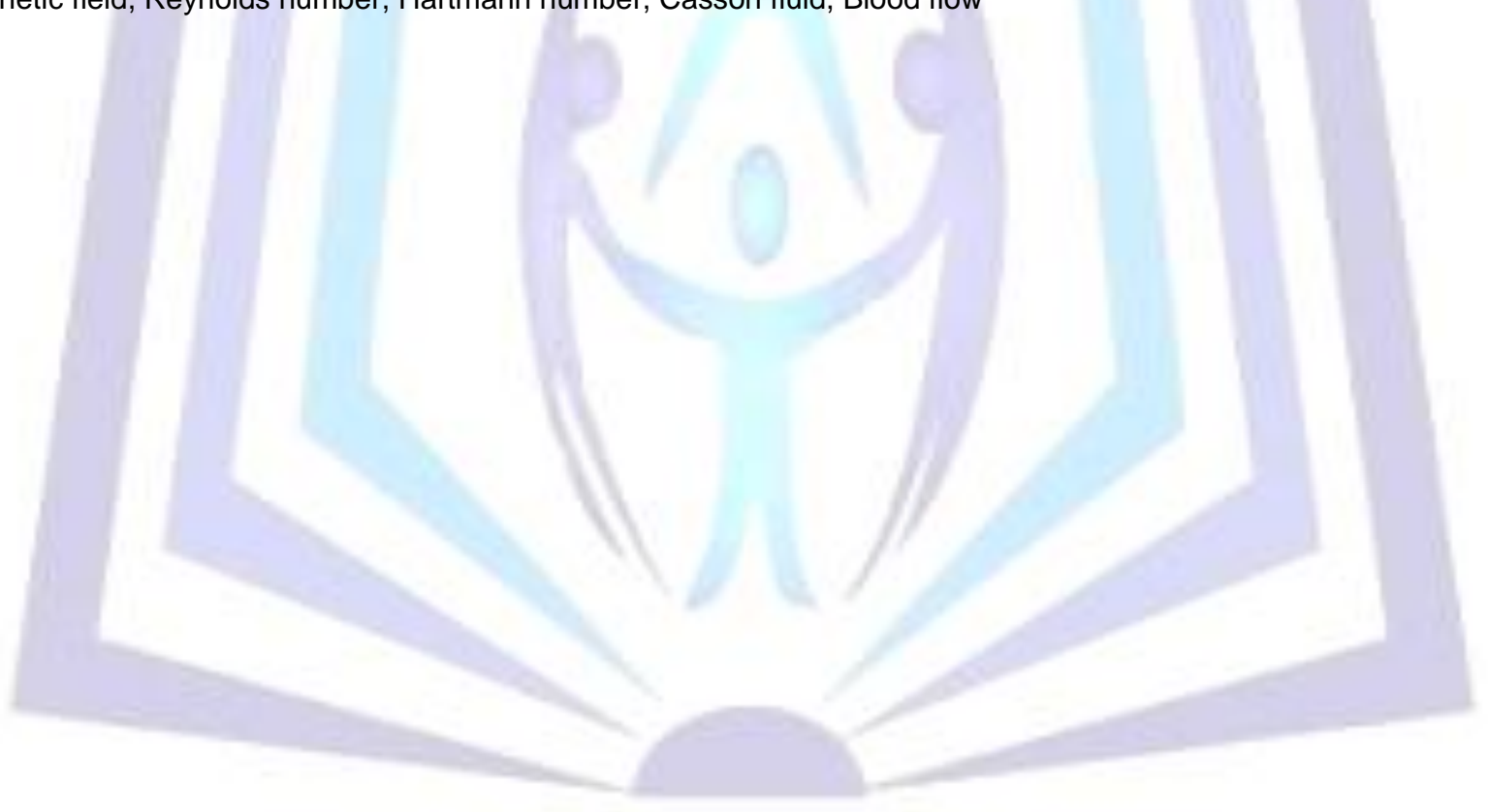

\section{Council for Innovative Research}

Peer Review Research Publishing System

Journal: International Journal of Management \& Information Technology

Vol.4, No.2

editor@cirworld.com

www.cirworld.com, member.cirworld.com 


\section{INTRODUCTION}

It is well known that non-Newtonian nature of blood significantly influences the flows, particularly in the cases where blood vessels are curved, branching or narrow etc. Stenosis refers to localized narrowing of an artery and is a frequent result of arterial disease and is caused mainly due to intravascular atherosclerotic plaque which develops at the arterial wall and protrudes into the lumen of the vessel. Such constrictions disturb normal blood flow through the artery. There is considerable evidence that hydrodynamic factors can play a significant role in the development and progression of this disease. Several flow characteristics, such as wall shearing stress (Caro [31]), pressure (Taxon [181]) and turbulence (Wesolowski [190]) may have potential medical significance. Higher resistance to flow can also become increasingly important as the stenosis becomes more severe (May [111]). Although the potential importance of hydrodynamic factors has been recognized for many years, the study of the mechanics of flow in constricted tubes remains a formidable problem. To study the detail the flow in a stenosis Young [200] considered the flow in a middy constricted tube based on a highly simplified linear model which work extended by Forrester and Young [77] to include the effects of flow separation on a mild constriction. The flow of blood in human circulatory system can be controlled by applying appropriate magnetic field. Many researchers have shown that blood is an electrically conducting tube. The Lorentz's force will act on the constituent particles of blood and this force will oppose the motion of the blood and thus reduces its velocity. This decelerated blood flow may help in the treatment of certain cardiovascular diseases and in the diseases with accelerated blood circulations such as hypertension, hemorrhages etc. Many works have been done in this field. Rathod and Gayatri [140] studied the conducting pulsatile blood flow with micro organisms represented by two fluid model through circular channel which diverges at a slow exponential rate under the magnetic field. It is suggested by Karchevski and Marochnik [99] that there is possibility of regulating moment of blood by application of an external magnetic field. It has been observed by many investigators that by the application of an external magnetic field, the biological systems are greatly affected. The effect of static magnetic field on the blood flow is studied by Suri and Puspa [177]. Ramachandra Rao Deshi kachar [137] have given an excellent review of a good number of works concerning the effect of a magnetic field on flow characteristic of blood.

Morgan and Young [118] and Shukla [160] studied blood flow in stenosed artery without magnetic effect. They have considered no slip at the boundary. It is observed that under certain conditions, there exits a slip at the wall in blood flow. Effects of slip in blood flow through stenosed artery has been studied by Chaturani and Biswas [41] without magnetic effect. Bhuyan and Hazarika [11] have studied its effect with magnetic effect. Although at high shear rates and in large diameter arises, blood behaves like a Newtonian fluid, but it is not so in narrow tubes. It has been observed that blood being a suspension of red cells in plasma (a Newtonian fluid) behaves like a non Newtonian fluid at low shear rate in smaller diameter tubes. Here an attempt is made to study the flow of blood through a stenosed artery with the effect of slip at the boundary in presence of transverse magnetic field considering blood as non- Newtonian fluid.

\section{MATHEMATICAL FORMULATION OF THE PROBLEM}

Steady laminar flow of blood through an axially symmetric stenosed artery in presence of a transverse magnetic field is considered (Fig. 1). The axial coordinate and velocity are $\hat{z}$ and $\hat{u}$ respectively, and are considered to be positive in the downstream direction with $\hat{U}$ as centerline velocity. The radial coordinate $\hat{r}$ and velocity $\hat{v}$ are positive when directed outward from the centerline. The local radius of the axi symmetric tube is $\hat{R}(\hat{z})$ and $R_{0}$ is the radius of the unconstricted sections in the upstream and downstream of the stenosis, $B$ is the applied magnetic field in $\hat{r}$ direction. The equations that govern the flow, under the assumed conditions are the continuity equation and the NavierStokes equation. Under the magnetic field applied in $\hat{r}$ direction the equations in the axial and radial directions in dimensionless form become,

$$
\begin{aligned}
& \frac{\partial u}{\partial z}+\frac{\partial v}{\partial r}+\frac{v}{r}=0 \\
& u \frac{\partial u}{\partial z}+v \frac{\partial u}{\partial r}=-\frac{\partial P}{\partial z}+\frac{2}{\operatorname{Re}}\left[\frac{\partial^{2} u}{\partial r^{2}}+\frac{1}{r} \frac{\partial u}{\partial r}+\frac{\partial^{2} u}{\partial z^{2}}\right]-\frac{2}{\operatorname{Re}} M^{2} u \\
& v \frac{\partial v}{\partial r}+u \frac{\partial v}{\partial z}=-\frac{\partial P}{\partial r}+\frac{2}{\operatorname{Re}}\left[\frac{\partial^{2} v}{\partial r^{2}}+\frac{1}{r} \frac{\partial v}{\partial r}-\frac{v}{r^{2}}+\frac{\partial^{2} v}{\partial z^{2}}\right]
\end{aligned}
$$


Where $M=\sqrt{\frac{\sigma}{\rho v}} B R_{0}=$ Hartmann number

$$
\operatorname{Re}=\frac{2 R_{0} U_{0}}{v}=\frac{2 R_{0} U_{0} \rho}{\mu}=\text { Reynolds number, } \mu \text { is the fluid viscosity }
$$

In order to proceed, three simplifying assumptions are made.

(i) The terms due to the viscous component of the normal stress in the axial direction $\left[\frac{\partial^{2} u}{\partial z^{2}}\right]$ are negligible which has been extensively used in the analysis of non-uniform flow.

(ii) The axial velocity is expressible as a suitable polynomial.

(iii) The pressure gradient term can be eliminated since,

$$
\int_{0}^{R} r \frac{\partial P}{\partial z} d r=R^{2} \int_{0}^{R} r u \frac{\partial P}{\partial z} d r
$$

Boundary conditions are

(i) $u=u_{s}$ at $\mathrm{r}=\mathrm{R}(\mathrm{z})$

(ii) $u=U(z), \frac{\partial u}{\partial r}=0, \frac{\partial^{3} u}{\partial r^{3}}=0$ at $r=0$

(iii) $\int_{0}^{R}(r u) d r=\frac{1}{2}$

(iv) $\mathrm{U}=\mathrm{U}$ and $\frac{\partial u}{\partial r}=0$ at $\frac{r}{R}=\lambda$

Multiplying (2) by $r$, rearranging and integrating over the cross section of the tube, we get the integral momentum equation. In the same manner, an integral energy equation is obtained by multiplying equation (2) by ru and integrating over the cross section. Using the above assumptions and combining the integral momentum and integral energy equations we get,

$$
\begin{array}{r}
\frac{1}{2} R^{2} \frac{\partial}{\partial z} \int_{0}^{R} r u^{3} d r-\frac{\partial}{\partial z} \int_{0}^{R} r u^{2} d r=-\frac{2}{\operatorname{Re}}\left[R^{2} \int r\left(\frac{\partial u}{\partial r}\right)^{2} d r+R\left(1-R^{2} u_{S}\right)\left(\frac{\partial u}{\partial r}\right)_{R}\right] \\
-\frac{2 M^{2}}{\operatorname{Re}}\left[R^{2} \int_{0}^{R} u r^{2} d r-\int_{0}^{R} u r d r\right]
\end{array}
$$

The dimensionless polynomial velocity profile can be expressed as

$$
\frac{u}{U}=A+B(r / R)+C(r / R)^{2}+D(r / R)^{3}+E(r / R)^{4}
$$

On using the boundary conditions (i) to (iii) we get

$$
u=R^{-2}\left[F+2(3-2 F-G)(r / R)^{2}-3(2-F-G)(r / R)^{4}\right]
$$

Where $F=R^{2} U, G=R^{2} u_{s}, u_{s}$ is slip velocity, when $F=2$ and $u_{s}=0$ in (6) parabolic profile is obtained. The blunted profile can be expressed as

$$
u=\left\{\begin{array}{cc}
U & 0 \leq r / R \leq \lambda \\
a+b(r / R)^{2}+C(r / R)^{4}, & \lambda<r / R \leq 1
\end{array}\right.
$$

Using the boundary condition (iv), equation (7) reduces to 
$u=\left\{\begin{array}{cc}U & 0 \leq r / R \leq \lambda \\ u_{s}+\frac{U-u_{s}}{\left(1-\lambda^{2}\right)^{2}}\left[1-2 \lambda^{2}+2 \lambda^{2}(r / R)^{2}-(r / R)^{4}\right] & 0<r / R \leq 1\end{array}\right.$

Where $\lambda=\sqrt{\frac{(3-2 F-G)}{(F-G)}}$

When $2 \mathrm{~F}+\mathrm{G}=3, \lambda=0$ and with this restriction the blunted velocity profile equation (7) reduces to a polynomial profile. When $2 F+G<3, \lambda$ is positive and real. When $2 F+G>3, \lambda$ is imaginary. So, the blunted velocity profile, equation (7) will be valid for $2 \mathrm{~F}+\mathrm{G}<3$ and for the case $2 \mathrm{~F}+\mathrm{G} \geq 3$, the polynomial velocity profile equation (6) will be used to approximate the flow.

The profile equations (6) and (8) are substituted into (4). Then it becomes

$$
\begin{gathered}
U^{\prime}=\left[-R^{-3} R^{\prime}\left\{0.0286\left(F^{3}+G^{3}\right)-0.1333\left(F^{2}+G^{2}\right)+0.2571(F+G)+F G(0.0667-\right.\right. \\
\left.0.0143(F+G)-0.3429\}+4\left(U-u_{S}\right) \Gamma / \operatorname{Re}-2 M^{2} \Gamma_{1} / \operatorname{Re}\right]\left[0.0429 F^{2}-0.0048 F+\right. \\
G(0.0119-0.0143 F-0.0071 G)-0.028]^{-1}
\end{gathered}
$$

where,

$$
\begin{gathered}
\Gamma=\left\{\begin{array}{cc}
\frac{(G-F)}{3(F-1)}\left\{\frac{(F-G)^{2}\left(43 F^{2}+G^{2}-12 G+10 F G-96 F+54\right)+2 \times 9^{2}(F-1)^{3}(G-1)}{9^{2}(F-1)^{3}}\right\} & 2 F+G \geq 3 \\
\Gamma_{1} & =\left\{\begin{array}{cc}
{[R 3\{F(-0.072)+G(0.028)-0.737\}-0.5]} & 2 F+G \geq 3 \\
G\left(0.333 R^{3}-0.5\right)+\frac{(F-G)^{2}}{9(F-1)^{2}}\left\{F\left(4.058 R^{3}-1.333\right)+G\left(0.742 R^{3}-0.166\right)-\left(4.8 R^{3}-1.5\right)\right. & 2 F+G<3
\end{array}\right.
\end{array}\right.
\end{gathered}
$$

The primed quantities represent derivatives in the axial direction. The initial condition for solving (12) comes from the asumptio of poiseuille flow in far upstream of the stenosis, i.e., $z=-\alpha, R=1, U=2$.

(9)

The shear stress component at any distant $r$ from the tube axis is given by

$$
\begin{aligned}
\tau_{r z}=\mu \frac{d u}{d r} & \\
& =\left\{\begin{array}{cc}
\frac{\mu z_{0} U^{\prime}}{4 \pi} \frac{1}{\sin \frac{\pi}{z_{0}}} & 0 \leq r / R \leq \lambda \\
\mu\left(\frac{U-u_{s}}{1-\lambda^{2}}\right)\left[\frac{4 \lambda^{2}}{R}\left(\frac{r}{R}\right)-\frac{4}{R}\left(\frac{r}{R}\right)^{3}\right], & \lambda<r / R \leq 1
\end{array}\right.
\end{aligned}
$$

Expression for wall shear stress $\left(\tau_{w}\right)$ can be obtained from the formula,

$\tau_{w}=\left.\tau_{r z}\right|_{r=R}$ 


$$
=\left\{\begin{array}{cc}
\frac{\mu z_{0} U^{\prime}}{4 \pi} \frac{1}{\sin \frac{\pi z}{z_{0}}} & 0 \leq r / R \leq \lambda \\
\frac{\mu z_{0}}{4 \pi} \frac{1}{\sin \frac{\pi z}{z_{0}}\left(\frac{U-u_{s}}{1-\lambda^{2}}\right) \frac{r^{2}}{R^{3}}\left[-4 \lambda^{2}+\frac{r^{2}}{R^{2}}\right],} & \lambda<r / R \leq 1
\end{array}\right.
$$

Also under high shear blood behaves as Newtonian fluid and in the neighborhood of the blood vessel wall, shear rate is high enough for the Newtonian assumption to hold good for normal blood. Also, toward the centre of the tube, the shear gradient tends to zero and the non-Newtonian behaviour will become prominent. Further, it is reported that blood has a finite yield stress $\left(\tau_{y}\right)$. From the above considerations, Cassson behavior of blood is found evident. At tube wall ( $\left.r=R\right)$, the shear stress is $\tau_{w}$ and at a certain distance from the tube axis i.e., along the stress axis, it is $\tau_{y}$, the yield stress which correspondence on the horizontal axis is $r_{c}$, a critical radius (Fig 2). Thus their arises two cases viz., (i) if the shear stress $\tau_{r z}$ at a distance $r$, is not higher than he yield stress $\tau_{y}$, i.e., $\tau_{r z} \leq \tau_{y}$ or $r \leq r_{c}$, blood will not follow and (ii) if shear stress is not lower than its yield value i.e., $\tau_{r z} \geq \tau_{y}$ or $r \geq r_{c}$, blood flow will be possible. Using the above equation (14) and $\tau_{r z}\left(r_{c}\right)=\tau_{y}$, the expression for $\tau_{y}$

$\tau_{y}=\left.\tau_{r z}\right|_{r=r_{c}}$

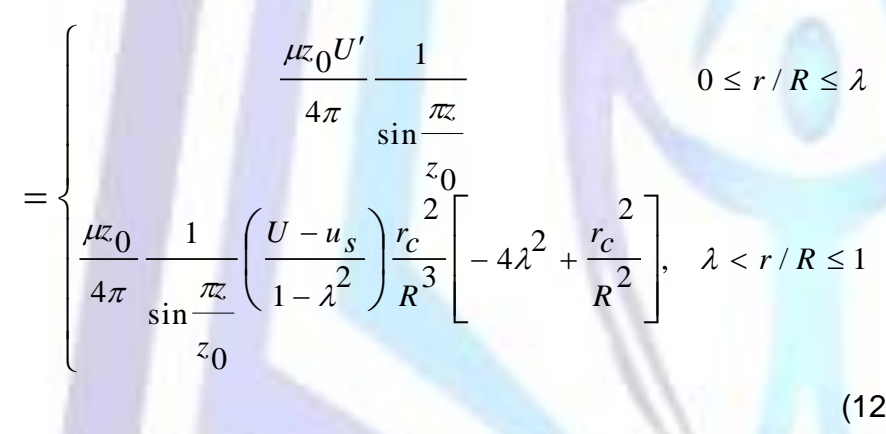

In between the stresses $\tau_{y}$ and $\tau_{w}$ there may arise two cases viz., wall shear stress greater and smaller than yield stress.

If $\tau_{y} \geq \tau_{w}$ i.e., if $r_{c} \geq R$ then no flow will occur, accordingly velocity function will result to

$u=0$

And if $\tau_{y} \leq \tau_{w}$ i.e., if $r_{c} \leq R$ then there will be flow and for that

$u=u(r)$

Again, Casson's equation may be reproduced in the following form:

$$
\begin{array}{rlrl}
\dot{\gamma}=f\left(\tau_{r z}\right) & =\frac{1}{K_{c}}\left(\sqrt{\tau_{r z}}-\sqrt{\tau_{y}}\right)^{2}, \tau_{r z} \geq \tau_{y} \\
& =0 & , \tau_{r z} \leq \tau_{y}
\end{array}
$$


Now, the vanishing of the strain rate, i.e., $\dot{\gamma}=0$, implies that $\frac{d v}{d r}=0$, which on integration results to

$$
\mathrm{V}=\mathrm{constant}=\mathrm{v}_{\mathrm{c}} \text { when } \quad \tau_{r z}=\tau_{y}
$$

Where $v_{c}$ is the core velocity at $r=r_{c}$ (core radius).

Thus for blood flow when $r_{c}<R$ there will be two regions viz., $0 \leq r \leq r_{c}$ and $r_{c} \leq r \leq R$ and it is, obvious, for the region between 0 and $r_{c}$, equation representing the flow is

$\frac{d v}{d r}=0, \quad 0 \leq r \leq r_{c}$

which on integration provides $\mathrm{v}=\mathrm{v}_{\mathrm{c}}$ for $0 \leq r \leq r_{c}$ indicating that velocity profile will be flat and for $r_{c} \leq r \leq R, u$ will show deviations from the flat profile and Casson's equation has to be applied for this domain of blood flow. From this question, it is easily found that

$\frac{d v}{d r}=\frac{1}{K_{c}}\left[\sqrt{\tau_{r z}}-\sqrt{\tau_{y}}\right]^{2}, r_{c} \leq r \leq R$

Which is the governing equation of the fluid flow and it has been integrated with the introduction of a velocity slip condition at the vessel wall.

\section{SOLUTION OF THE PROBLEM}

Using the slip velocity condition at the vessel wall

$\mathrm{v}=\mathrm{v}_{\mathrm{S}}$ at $\mathrm{r}=\mathrm{R}$

Where $v_{s}$ is a constant slip velocity at tube wall in the axial direction, equation (19) is integrated between $r$ and $R$ numerically.

The solution of the equation is solved by numerically.

From the above expression and others consideration velocity distribution $V_{z}$ can be re written in the following style

$v=\left[\begin{array}{cc}v(r) & r_{c} \leq r \leq R \\ v_{c} & 0 \leq r \leq r_{c} \\ 0 & r_{c}>R\end{array}\right.$

Also the rate of volume flow can be obtained from

$Q=2 \pi \int_{r=0}^{r=R} r v d r$

\section{Young's model:}

The particular stenosis geometry used was selected after Young $\left[{ }^{\star}\right]$ and is described by the expressions

$R(z)=\left\{\begin{array}{cl}1-\frac{\delta}{2}\left(1+\cos \frac{\pi z}{Z_{0}}\right) & ,|z| \leq Z_{0} \\ 1 & ,|z|>Z_{0}\end{array}\right.$ 
where $\delta=($ Stenosis height $) / \mathrm{R}_{0}$ and $\mathrm{Z}_{0}=($ Stenosis length $) / 2 \mathrm{R}_{0}$. Three model stenosis with geometries defined in the above, but with different values of $\delta$ and $\mathrm{Z}_{0}$ were constructed for the experimental testes (Table 1).

Table 1

Geometric parameters of the model Stenosis

\begin{tabular}{|l|l|l|l|l|}
\hline $\begin{array}{l}\text { Model } \\
\text { Numbers }\end{array}$ & $\mathrm{R}_{0}$ & $\delta$ & $\mathrm{Z}_{0}$ & Percent Stenosis \\
\hline $\mathrm{M}-1$ & 0.372 & $1 / 3$ & 4 & 56 \\
\hline $\mathrm{M}-2$ & 0.372 & $2 / 3$ & 4 & 89 \\
\hline $\mathrm{M}-3$ & 0.372 & $2 / 3$ & 2 & 89 \\
\hline
\end{tabular}

For classification purposes the severity of a stenosis is often defined by the maximum reduction in lumen area expressed as a percentage (percent stenosis). Model M-1 represents a relatively mild stenosis with a 56 percent severity of constriction and a total length of $8 \mathrm{R}_{0}$. Model $\mathrm{M}-2$ has the same length but is more severe with 89 percent constriction. To evaluate the effect of stenosis length, model M-3 was designed to have the same severity as M-2 but only half of its length.

\section{RESULTS AND DISCUSSION}

The problem under investigation is solved numerically using Shooting Method. Numerical calculations have been done for various combinations of parameters i.e., the Hartmann number $\mathrm{M}$ and Reynolds number Re with slip and no slip. The Young model is used in computing the centerline velocity $U$. After obtaining the centerline velocity $U$ from (8), the axial velocity profiles $(u)$ are computed directly by substituting $U$ into $(5)$ or (7). Then the value of $u$ substituting in equation (19) the velocity profile $v$ are computed. The wall shear stress $T_{w}$ has been computed from the relation (11) for both slip and no slip case.

Numerical results are shown graphically. It has been observed that the effect of the Hartmann number M and Reynolds number Re on the velocity field as well as on the wall shear stress is very prominent.

It is observed that the centerline velocity $U$ decreases with increasing Reynolds number at $M=1.5$ (Fig. 3). The nature of $U$ is also same with no slip (Fig. 4). The centerline velocity $U$ increases when the Hartmann number increases with slip at $R e=50$ (Fig. 1). The nature of $U$ is same in no slip case also (Fig. 2). It is seen that when Hartmann number increases the axial velocity (v) decreases with slip (Fig. 5). A similar case occurs in no slip also (Fig. 6). The axial velocity increases when Reynolds number increases with slip (Fig. 7). A similar case occurs in no slip also (Fig. 8).

The present problem reduces to the problem discussed by Chaturani $\left[{ }^{\star}\right]$ when $M=0$. It is observed that when the Hartmann number increases the fluid velocity and the wall shear stress is greatly affected. Numerical results agree well with their solution in non-magnetic case. The mathematical expressions may help medical practitioners to control the blood flow of a patient whose blood pressure is very high, by applying certain magnetic field.

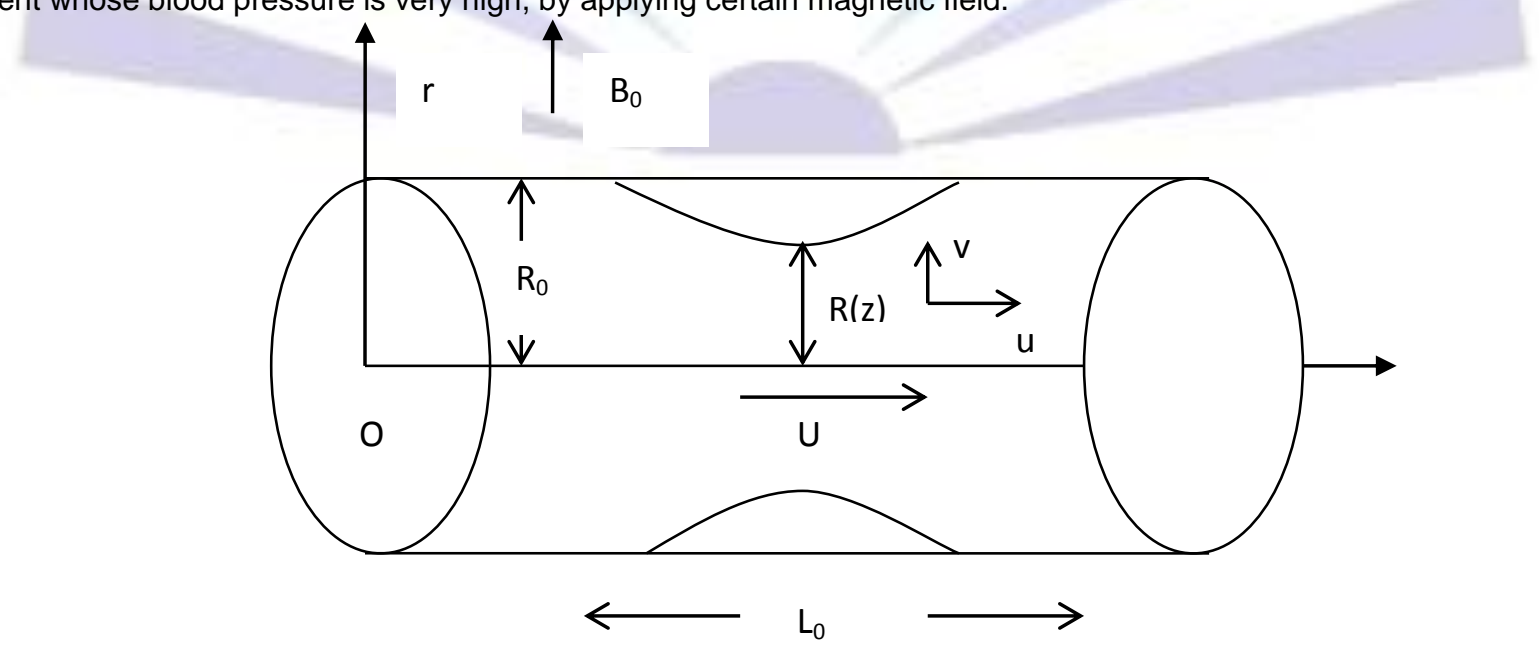

Fig. 1. Geometry of an arterial stenosis with axial velocity slip at wall. 


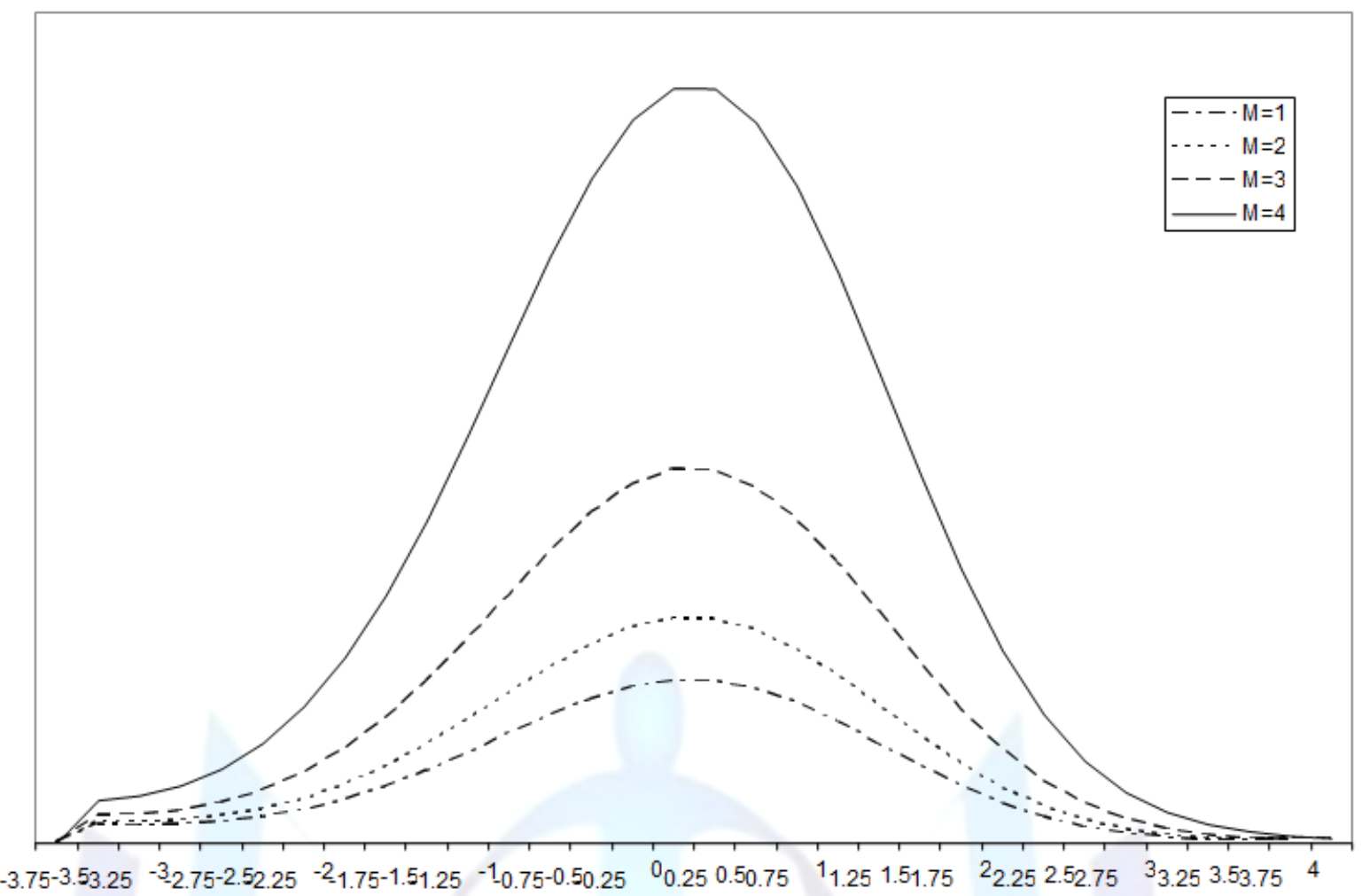

Fig. 1. Variation of Center line Velocity for different Hartmann number at Re=50

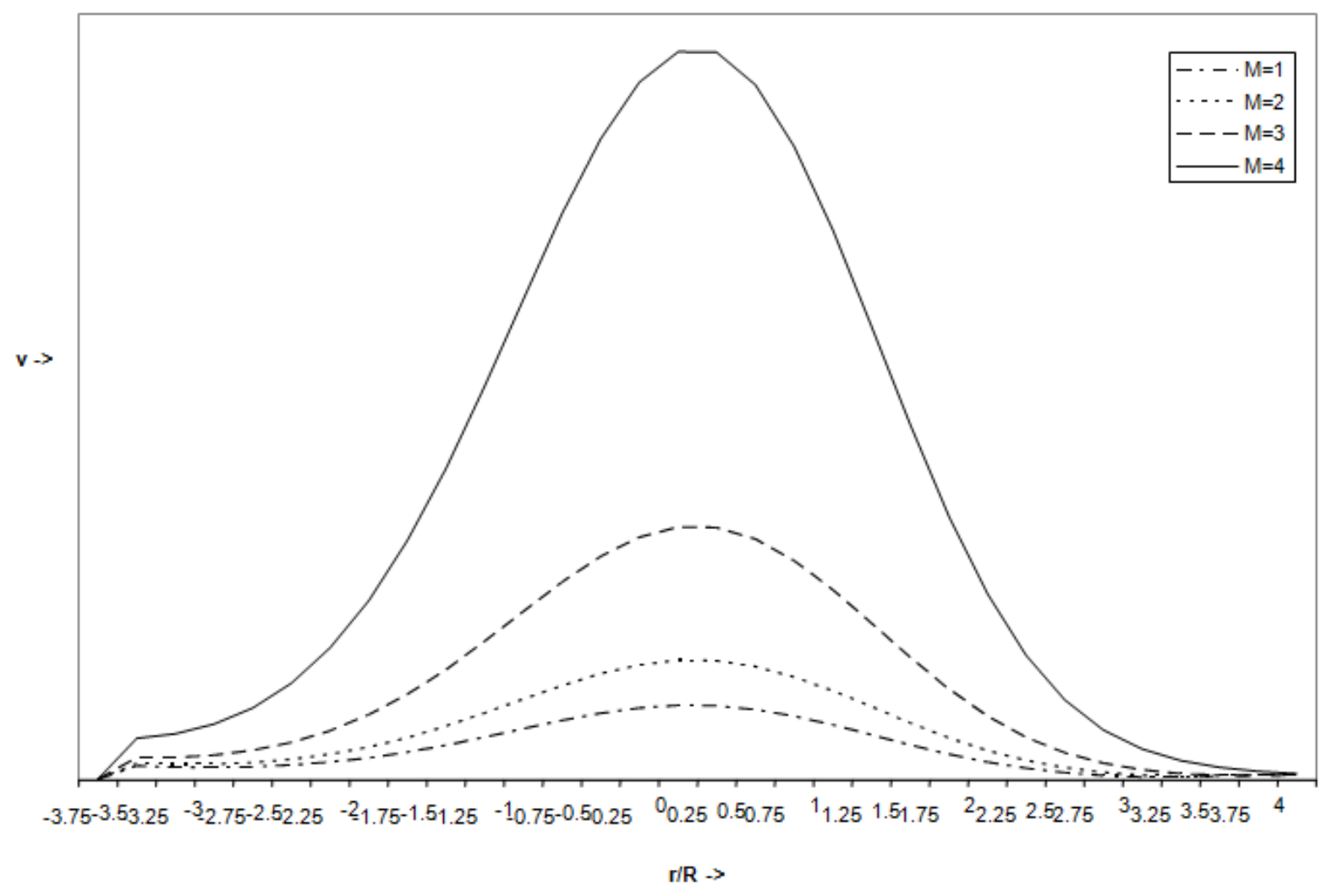

Fig. 2. Variation of Center line velocity for different Hartmann number at $\operatorname{Re}=60$ with no slip 


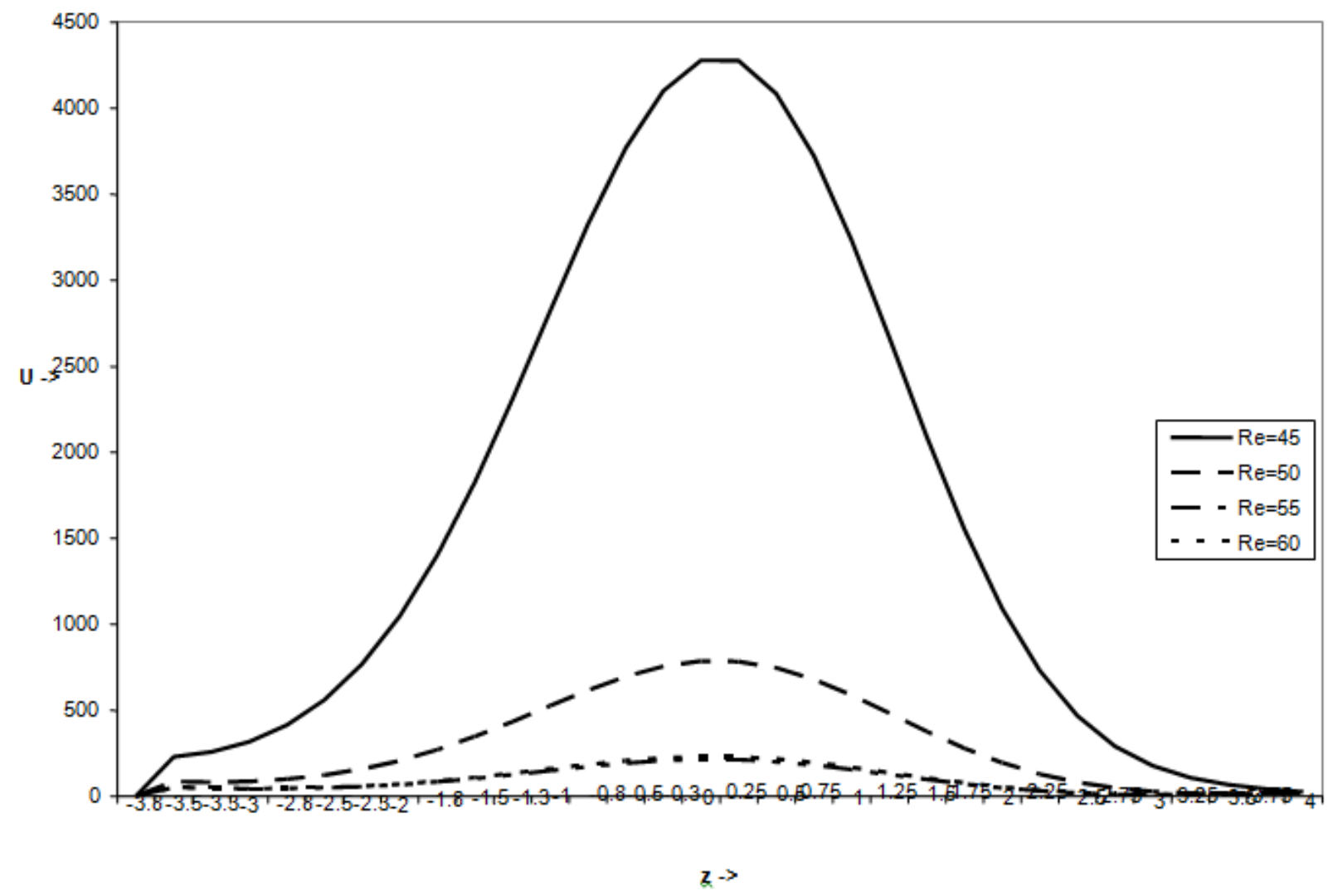

Fig. 3. Variation of center line velocity for different Reynolds numbers at $\mathrm{M}=1.5$ with slin

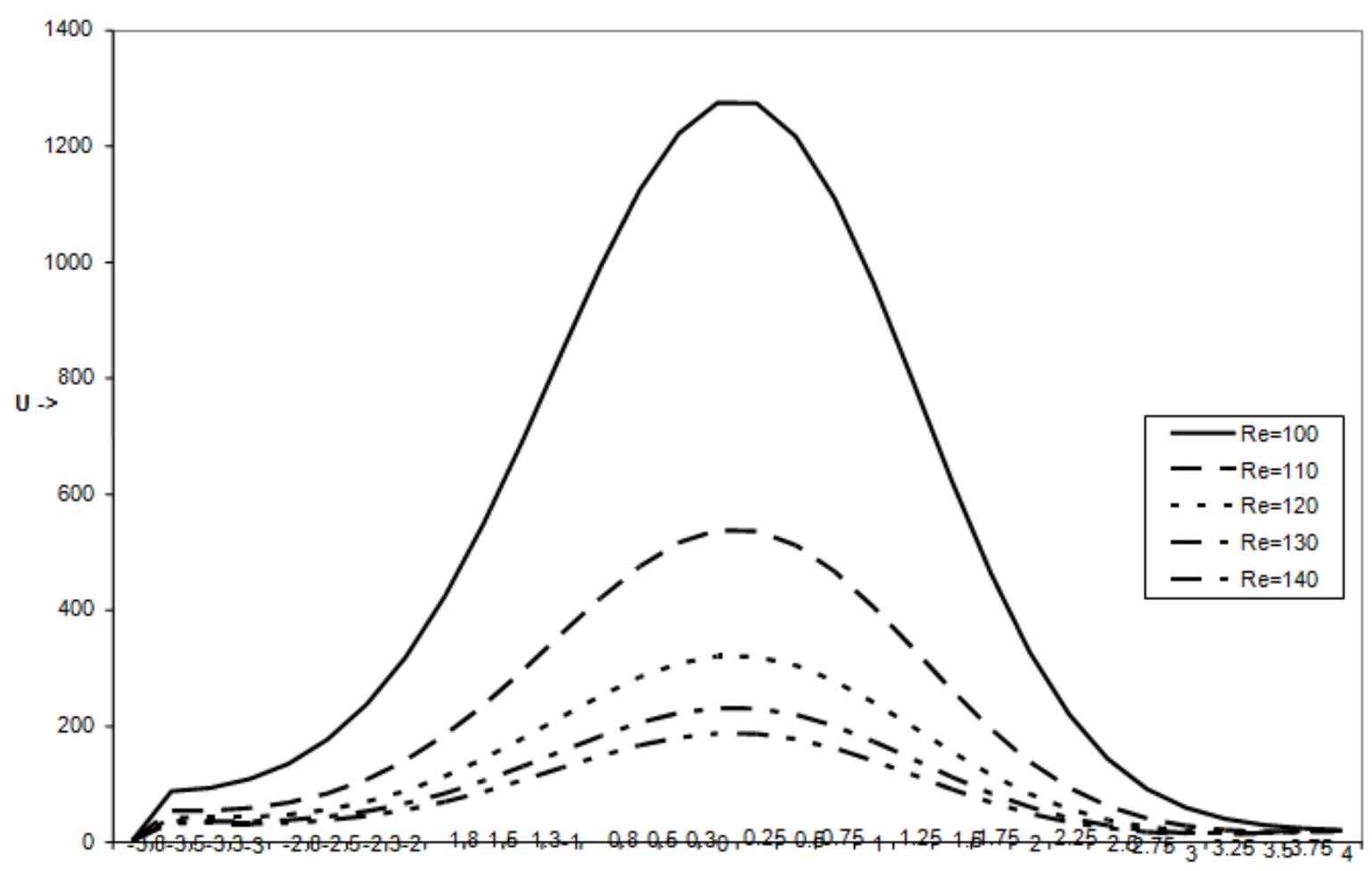

$z \rightarrow$

Fig. 4. Variation of center line velocity for different Reynolds numbers at $M=1.5$ with no slip 


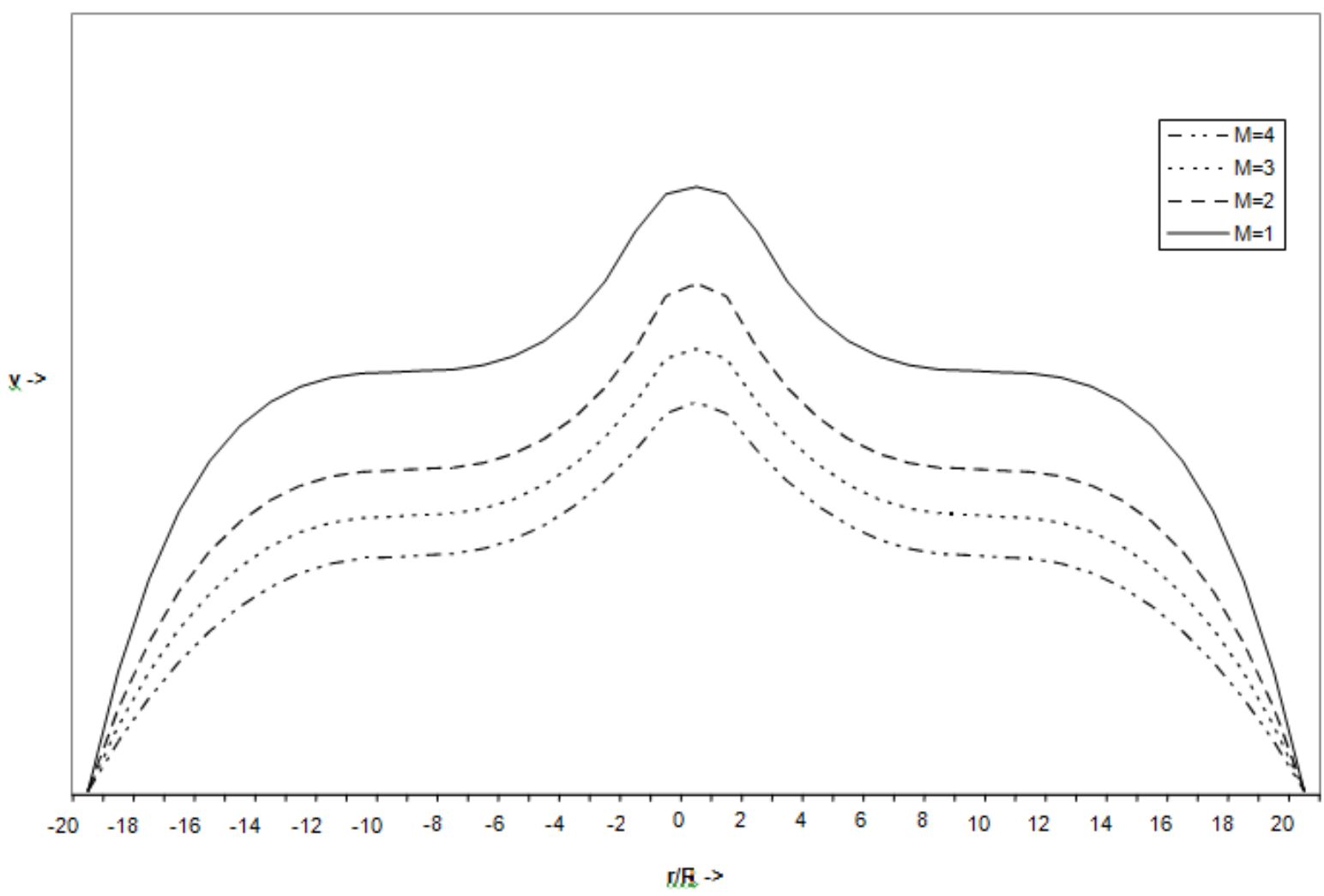

Fig. 5. Variation of Velocity profile for different Hartmann numbers at $\operatorname{Re}=\mathbf{5 0}$ with slip

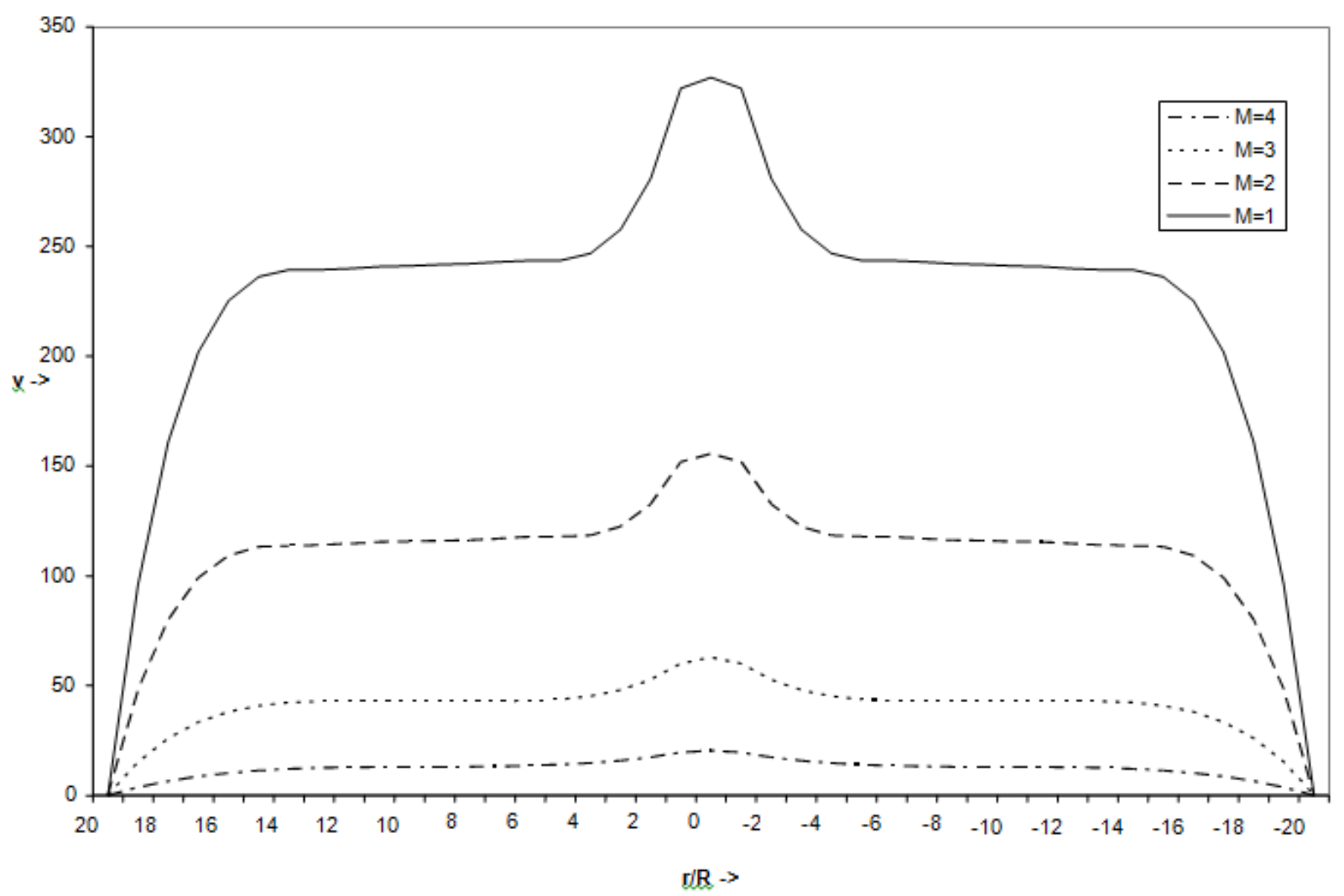

Fig. 6. Variation of Velocity profile for different Hartmann number at $\mathrm{Re}=50$ with no slip 


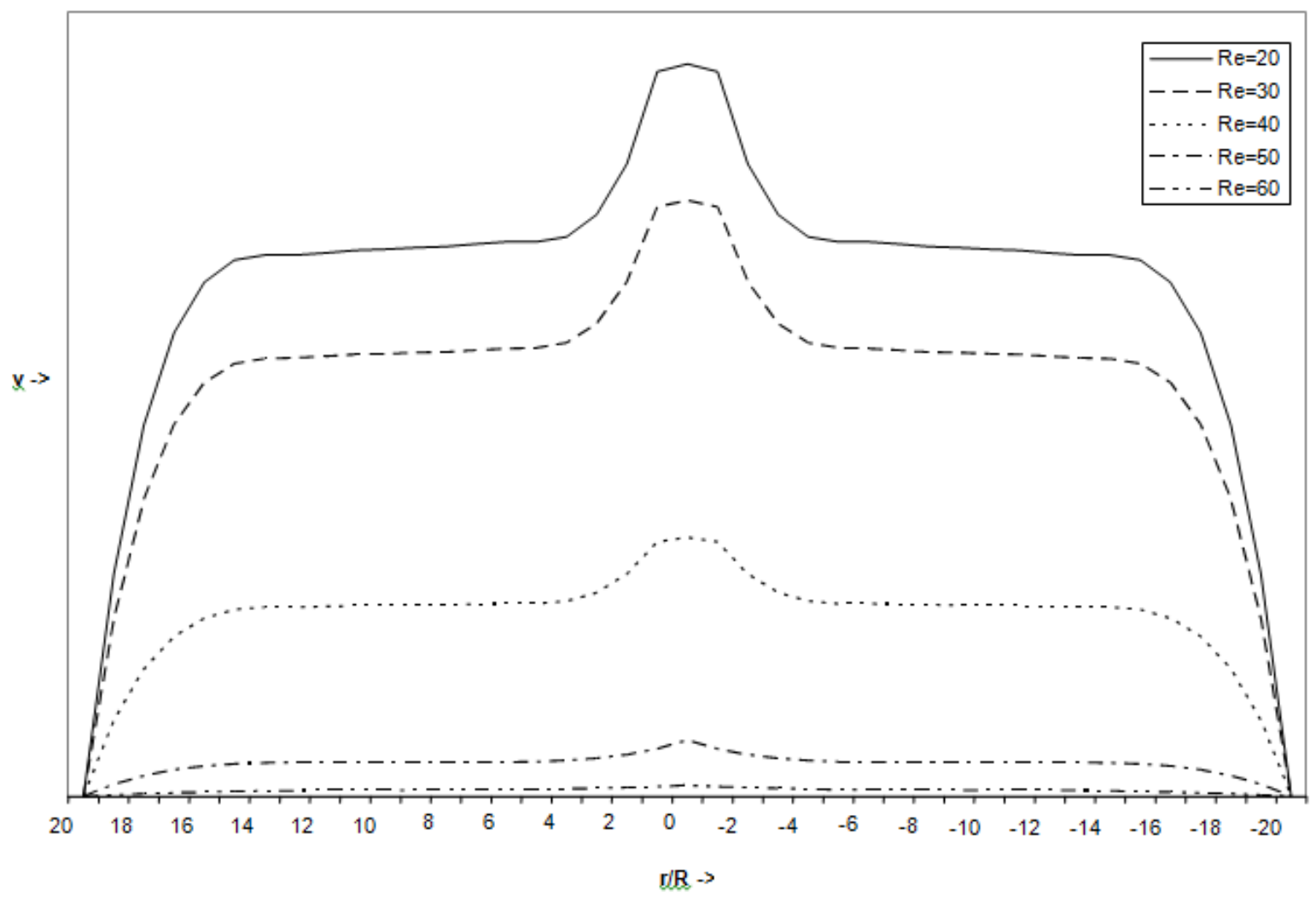

Fig. 7. Variation of Velocity profile for different Reynolds number at $M=0.50$ with slip

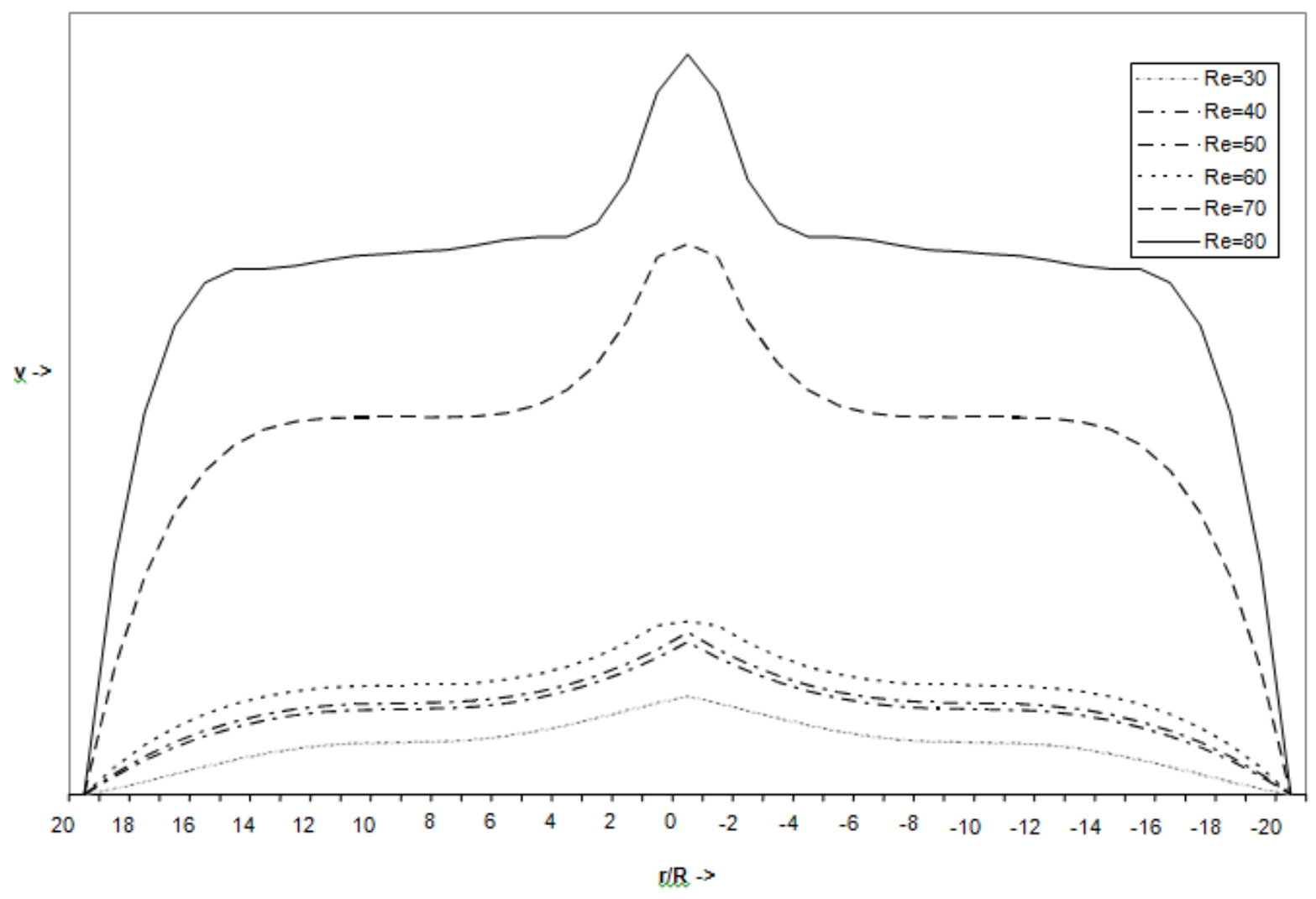

Fig. 8. Variation of Velocity profile for different Reynolds number at $M=0.50$ with no slip 


\section{REFERENCES}

1. Caro, C.G., Arterial Fluid Mechanics and Atherogenesis : Recent Advances in Cardiovascular Disease, ed. Niimi, H., vol. Il (Supplement), Suita, Osaka, Japan, pp. 7-11 (1981)

2. Forrester, J.H. and Young D.F., Flow Through a Converging Diverging Tube and its Applications in Oclusive Vascular Disease-I, J. Biomech., 3, pp. 297-306 (1970).

3. Korchevski, E.M. and Marochnik, L.S., Biofizika, Vol. 10, pp. pp. 371-373 (1965).

4. May, A.G., Deweese, J.A. and Rob, C.G., Hamodynamics effect of arterial stenosis, Surgery, Vol. 53, pp. 513524

5. Morgan, D.E. and Young D.F., An Integral Methods for the Analysis of Flow in Arterial Stenosis, Bull. Math. Biol. 36, pp. 39-53 (1974).

6. Ramachandra Rao, A. and Desikachar, K.S., J.Indian Inst. Sc., Vol. 68, pp. 247-260 (1988)

7. Rathod, V.P., Gayatri, Applied Science Periodical, Vol. 2 (1), pp. 52-58 (2000)

8. Shukla, J.B., Parihar, R.S. and Gupta, S.P., Biorheology, Vol. 42, pp. 403 (1980).

9. Suri, P.K. and Puspa, R. Suri, I.J. of Pure Appl. Math., Vol. 12 (7), pp. 907-918, (1981)

10. Texon, M., Arch. Int. Med., Vol. 99, pp. 418 (1957)

11. Wesolowski, S.A., Fries, C.C., Sabina, A.M. and Sawyer, P.N., Mechanisms in Vascular Homeostasis and Intravascular Throbosis, Sawyer, Ed. Newyork: Appleton Century Croft. (1965)

12. Young, D.F., Fluid Mechanics of Arterial Steonses, J. Biomechanical Engng. Trans. ASME, 101, pp. 157-175 (1979). 